\title{
Detecção de carboidratos de baixo peso molecular em diferentes variedades de manga, em dois estágios de maturação
}

\author{
Detection of low molecular weight carbohydrates in different mango varieties at two stages of \\ maturation
}

\section{Detección de carbohidratos de bajo peso molecular en diferentes variedades de mango en dos etapas} de maduración

\section{Resumo}

O objetivo foi analisar o potencial hiperglicêmico de diferentes variedades de manga nos diferentes estágios de maturação. Pesquisa do tipo transversal, descritivo experimental de abordagem quantitativa. A aquisição das amostras foi realizada na central de abastecimento do Estado do Piauí. Utilizadas variedades de mangas Palmer, Rosa e Tommy Atkins em dois estágios de maturação, estado de maturação verde e madura. As amostras foram submetidas a procedimentos de análise em quintuplicata quanto ao teor de açucares de baixo peso molecular e umidade. As comparações entre mangas de diferentes variedades e estados de maturação tiveram resultado estatisticamente significativas ( $\mathrm{p}=0.0084)$, quando comparado com a manga Rosa madura. $O$ kit para determinação de glicose mostrou-se eficaz para determina-la do que a curva de analítica. O valor da regressão linear (glicose) de 0.976 foi estatisticamente significativo e desenvolveu uma fórmula matemática que permite estimar qualquer concentração. Houve relação constante de glicose/frutose em diferentes frutas, e criou-se uma regressão linear que gerou uma equação de reta no qual a frutose pode ser estimada a partir do valor da glicose. Os valores de umidade não foram estatisticamente significativos e não interfere no nível de glicose. As variedades de manga testada apresentaram variações estatisticamente significativas nos teores de carboidrato de baixo peso molecular especialmente a variedade Rosa. O método enzimático desenvolvido demonstrou-se capaz para a quantificação espectrofotométrica do teor de glicose. É necessária a determinação do índice glicêmico para as variedades de frutas no Brasil.

Palavras-chave: Alimento; Fruta; Hiperglicemia; Índice glicêmico; Carga glicêmica.

\begin{abstract}
The objective was to analyze the hyperglycemic potential of different mango varieties at different stages of maturation. Cross-sectional, descriptive experimental research with a quantitative approach. Sample acquisition was performed at the supply center in the State of Piauí. Palmer, Rosa and Tommy Atkins mango varieties were used in two stages of ripeness, green and ripe state of ripeness. The samples were submitted to analysis procedures in quintuplicate for the content of low molecular weight sugars and moisture. Comparisons between mangoes of different varieties and maturity stages had statistically significant results $(\mathrm{p}=0.0084)$, when compared with ripe pink mango. The glucose determination kit proved to be effective in determining glucose than the analytical curve. The linear regression (glucose) value of 0.976 was statistically significant and developed a mathematical formula that allows estimating any concentration. There was a constant glucose/fructose relationship in different fruits, and a linear regression was created that generated a straight equation in which fructose can be estimated from the glucose value. Humidity values were not statistically significant and did not interfere with the glucose level. The tested mango varieties showed statistically significant variations in low molecular weight carbohydrate contents, especially the Rosa variety. The enzymatic method developed proved to be capable of spectrophotometrically quantifying the glucose content. It is necessary to determine the glycemic index for fruit varieties in Brazil.
\end{abstract}

Keywords: Food; Fruit; Hyperglycemia; Sugar level; Glycemic load. 


\begin{abstract}
Resumen
El objetivo fue analizar el potencial hiperglucémico de diferentes variedades de mango en diferentes etapas de maduración. Investigación experimental descriptiva transversal con enfoque cuantitativo. La adquisición de la muestra se realizó en el centro de abastecimiento del estado de Piauí. Las variedades de mango Palmer, Rosa y Tommy Atkins se utilizaron en dos estados de madurez, verde y maduro. Las muestras se sometieron a procedimientos de análisis por quintuplicado para el contenido de azúcares de bajo peso molecular y humedad. Las comparaciones entre mangos de diferentes variedades y etapas de madurez arrojaron resultados estadísticamente significativos $(\mathrm{p}=0,0084)$, en comparación con el mango rosado maduro. El kit de determinación de glucosa demostró ser eficaz para determinar la glucosa que la curva analítica. El valor de regresión lineal (glucosa) de 0.976 fue estadísticamente significativo y desarrolló una fórmula matemática que permite estimar cualquier concentración. Hubo una relación constante glucosa / fructosa en diferentes frutas, y se creó una regresión lineal que generó una ecuación directa en la que la fructosa se puede estimar a partir del valor de glucosa. Los valores de humedad no fueron estadísticamente significativos y no interfirieron con el nivel de glucosa. Las variedades de mango probadas mostraron variaciones estadísticamente significativas en el contenido de carbohidratos de bajo peso molecular, especialmente la variedad Rosa. El método enzimático desarrollado demostró ser capaz de cuantificar espectrofotométricamente el contenido de glucosa. Es necesario determinar el índice glucémico de las variedades de frutas en Brasil.
\end{abstract}

Palabras clave: Comida; Fruta; Hiperglucemia; Nivel de azucar; Carga glicemica.

\title{
1. Introdução
}

A manga (Mangifera indica L.) tem papel importante nas regiões tropicais por seu alto valor comercial e alimentício. Possui propriedades farmacológicas, efeitos antidiabéticos, antioxidantes, antialérgicas e anti-inflamatórias (Ganogpichayagrai, Palanuvej, Ruangrungsi, 2017). O Brasil é o sétimo produtor mundial de manga e, dentre as cultivares de importância comercial, a Tommy Atkins é a mais plantada e exportada (Marques et al., 2010). Esta juntamente com a variedade Rosa são as que possuem maior produção e consumo na região Nordeste brasileira (Melo, Araújo, 2011).

Os frutos da manga contém quantidades significativas de fibra, principalmente pectina, amido resistente e derivados de celulose e com alto teor de açúcar (frutose) (Maia et al., 2016). Durante o processo de amadurecimento, pode-se ocorrer alterações, como na firmeza da polpa, nos teores de açúcares, na coloração da casca e da polpa, na composição, teor de ácidos orgânicos e na perda de massa (Braz et al., 2008). É uma importante fonte de fitoquímicos, no qual se destaca os polifenóis, carotenóides e a vitamina C (Melo, Araújo, 2011).

Devido à constatação que diferentes alimentos contendo a mesma quantidade de carboidrato possuem distintos efeitos fisiológicos surge o conceito de índice glicêmico (Carvalho, Alfenas, 2008). A Carga Glicêmica (CG) é o produto do índice Glicêmico (IG)do alimento e o seu teor de carboidrato disponível. Dietas com cargas altas de IG e CG são rapidamente digeridas, absorvidas e transformadas em glicose. No entanto, dietas de baixo IG e CG proporcionam uma liberação de insulina e glicose lenta e gradual na corrente sanguínea (Silva et al., 2016). A estrutura do alimento pode afetar a resposta metabólica dos carboidratos e alimentos com baixo peso molecular (Lemos, Patek, Mezzomo, 2017).

Diante do exposto o objetivo da pesquisa foi analisar o potencial hiperglicêmico de diferentes variedades de manga nos diferentes estágios de maturação, através da determinação dos teores de carboidratos de baixo peso molecular das mangas selecionadas, Identificando os teores de umidade e sua relação com o potencial hiperglicêmico.

\section{Metodologia}

O presente estudo é do tipo transversal, descritivo experimental de abordagem quantitativa, que segundo Fontelles (2009), é toda pesquisa que envolve algum tipo de experimento, que visa observar, registrar e descrever as características de um determinado fenômeno, pesquisa quantitativa é aquela que trabalha com variáveis expressas sob a forma de dados numéricos e emprega rígidos recursos e técnicas estatísticas.

Foram utilizadas 3 variedades de manga sendo 5 unidades de cada variedade em dois estágios de maturação, verde e madura, totalizando 30 mangas. A aquisição das amostras foi realizada na véspera da análise, com exceção daquelas que 
sofrerão o processo de maturação para análise com base no estado de maturação. Foram utilizadas variedades de mangas Palmer, Rosa, Tommy Atkins. As análises foram realizadas em quintuplicata no laboratório de Química Geral do Centro Universitário Santo Agostinho. A metodologia descrita no manual do Instituto Adolfo Lutz (2008), com pequenas modificações, foi utilizada para análise de umidade e a análise de açúcares de baixo peso molecular foi realizada através do método espectrofotométrico utilizando o kit para determinação de glicose.

\subsection{Determinação de açúcares de baixo peso molecular}

O procedimento para análise de carboidratos foi direcionado para a determinação de carboidratos de baixo peso molecular. As amostras foram despolpadas, pesando $25 \mathrm{~g}$ e adicionou-se $100 \mathrm{ml}$ de água destilada, em seguida, triturou-se o material no liquidificador, submeteu-se ao processo de centrifugação para a separação das amostras, identificou-se e quantificou-se através da técnica para análise qualitativa pela técnica do açúcar redutor Benedict, pegou-seum alíquota desse material centrifugado e colocou-se nos tubos de ensaio e o Benedict o que reagiu tem açúcar redutor, e a técnica espectroscópica de quantificação de glicose para determinar quantitativamente a frutose presente, analisa no espectrofotômetro. Os tubos foram preparados pipetando-se 3,0ml do kit de glicose + 30 microlitros de cada solução, agitava-se as soluções, para homogeneizar, depois colocouse no aparelho de Banho Maria por 10 min, retirava e fazia a leitura da absorvância no espectrofotômetro da marca Quimis.A intensidade da coloração formada foi medida em espectrofotômetro no comprimento de onda $505 \mathrm{~nm}$.

\subsection{Curva de calibração}

Foi pesado em balança analítica $5 \mathrm{mg} / \mathrm{ml}$, ficando $500 \mathrm{mg}(0,5 \mathrm{~g})$ de glicose/frutose em um bécker e adicionou-se 20 $\mathrm{ml}$ de água destilada para dissolver totalmente o material, colocou-se no balão volumétrico de $100 \mathrm{ml}$ com o auxílio de um funil e logo após foi lavado o bécker e o bastão 3 vezes de $20 \mathrm{ml}$ de água destilada $(80 \mathrm{ml})$ para preparar uma solução mãe a $100 \mathrm{ml}$. A curva analítica de calibração foi construída considerando os seguintes volumes de glicose/frutose que foram diluídas na solução mãe completando com água destilada cada balão volumétrico de $10 \mathrm{ml}$, e obtendo as concentrações finais. Os tubos foram preparados pipetando-se $3,0 \mathrm{ml}$ do kit de glicose +30 microlitros de cada solução no balão volumétrico, agitou-se as soluções, para homogeneizar, depois colocou-se no aparelho de banho maria por $10 \mathrm{~min}$, retirava e fazia a leitura da absorvância em espectrofotômetrono comprimento de onda $505 \mathrm{~nm}$ da marca quimis, o que reagiu tem açúcar redutor. As concentrações utilizadas foram $0,1 \mathrm{ml} ; 0,5 \mathrm{ml} ; 1,00 \mathrm{ml} ; 2,00 \mathrm{ml} ; 3,00 \mathrm{ml} ; 4,00 \mathrm{ml} ; 5,00 \mathrm{ml}$. Sendo concentrações piloto por causa das amostras.

\subsection{Determinação da umidade}

A umidade foi determinada através do equipamento por utilização de radiação infravermelho, composto por uma balança que possui acoplada uma fonte da radiação. Para a realização da análise, cerca de $5 \mathrm{~g}$ da amostra foi aplicada a um suporte previamente tarado e a massa inicial foi registrada. Em seguida fez-se incidir a radiação sobre a amostra, e a massa final após o processo foi novamente registrada.

\subsection{Aspéctos éticos}

A pesquisa não está sujeita aos preceitos da resolução 466/2012 por não envolver direto ou indiretamente seres humanos. Os preceitos éticos relacionados à pesquisa científica foram respeitados, obedecendo-se as normas da Associação Brasileira de Normas Técnicas (ABNT). 


\subsection{Análise estatística}

Para a análises dos dados foram aplicados análise descritivas (média e desvio padrão), ANOVA e regressão linear, utilizando os softwares Prism 5.0 e Xlstat 2017.

\section{Resultados e Discussão}

\subsection{Carboidrato de Baixo Peso Molecular}

Carboidratos de baixo peso molecular são compostos basicamente por monossacarídeos, dissacarídeos e oligossacarídeos pequenos. Açúcares redutores são carboidratos que possuem carbono anomérico livre, capazes de se oxidar na presença de agentes oxidantes em solução alcalina (Santos et al, 2017).

Vale ressaltar que os teores de carboidrato de baixo peso molecular em diferentes alimentos podem variar de uma região para outra, tendo em vista que depende do estágio de maturação, condições de adubação do local de plantio, fatores climáticos e condições genéticas da planta. Nas frutas isso é mais intenso dependendo da fruta, a melancia por exemplo apresenta teores de glicose variando de 13,34 $\pm 3,154$, e frutose de 10,34 \pm 4,42 (Massa et al., 2014). Essa pesquisa utiliza o método enzimático para determinar carboidratos em frutas, no qual foi possível determinar a glicose e não os monossacarídeos totais.

Tabela 1. Média e desvio padrão dos açucares de baixo peso molecular (glicose) entre mangas de diferentes variedades e estados de maturação, Teresina, PI, 2018.

\begin{tabular}{ccc}
\hline Variedades & \multicolumn{2}{c}{ Maturação das frutas } \\
& Verde & Madura \\
Palmer & $1.10 \% \pm 0.54$ & $1.75 \% \pm 0.42$ \\
Tommy Atkins & $1.27 \% \pm 1.20$ & $2.08 \% \pm 0.65$ \\
Rosa & $3.65 \% \pm 0.45$ & $6.03 \% \pm 2.03$
\end{tabular}

$* \pm$ desvio padrão, teste estatístico ANOVA. $\mathrm{p}=0.0084$. Fonte: Dados da pesquisa (2018).

Como se observa na Tabela 1 entre as mangas de diferentes variedades e estados de maturação as diferenças entre estas comparações tiveram resultado estatisticamente significativos ( $\mathrm{p}=0.0084$.). Sendo que a manga rosa madura obteve maior média de 6.03 e a que obteve menor valor médio foi a Palmer verde 1.10 .

Observou-se uma quantidade de glicose maior ao encontrado nesta pesquisa durante o amadurecimento da manga Tommy atkins, com valores variando de 3,65 \% para 4,06 \% em frutas verdes e maduras respectivamente. O mesmo autor encontrou para a manga Rosa valor menor no estágio de maturação verde o teor de 2,15\% para açúcares redutores. Os frutos foram coletados na região da Chapada Diamantina no município de Piatã (BA) (Maciel, 2009).

Quanto aos açúcares redutores, os teores de 4,25\% na variedade Rosa e 4,10\% na Tommy Atkins constatou que as médias apresentaram entre si diferença estatisticamente significativa (Bezerra et al., 2011). Os conteúdos de açúcares redutores na polpa de manga Palmer madura foi 1,54\% para redutores, um valor maior do que a manga da pesquisa (Rocha, 2017). Ao estudarem cinco variedades de manga colhidas no Estado de São Paulo tendo a variedade Palmer apresentado 2,94\% para açúcares redutores (Silva et al., 2012). 
Vale ressaltar que a concentração de açúcares dos frutos depende das condições ambientais, pois excesso de água no estádio final de maturação pode resultar em frutos pouco doces, devido maior diluição dos açúcares (Massa et al., 2014). Os principais açúcares presentes nas frutas são a frutose e a glicose. No entanto, diferem significantemente em doçura. A informação sobre a quantidade de teores de glicose, composição (tipo e quantidade) são cruciais para os diabéticos (Caldas et al., 2015).

O consumo do açúcar em frutas in natura diariamente não parece estar relacionado com a ocorrência de doenças. No entanto, seu uso abusivo em sucos e alimentos, que contenham alta concentração de frutose, está diretamente associado com a incidência elevada de síndrome metabólica (Gaino, Silva, 2011).

Além da leitura direta dos resultados da concentração de glicose, também foi preparado uma curva de calibração utilizando glicose e o próprio kit. O método desenvolvido demonstrou-se capaz de quantificar glicose em amostras de mangas. O método desenvolvido demonstrou-se capaz de quantificar glicose em amostras de mangas. Esse método é específico para o analito glicose, pois se trata de um método enzimático, o qual apresenta alta seletividade, no qual está demonstrado no Gráfico 1 a seguir.

Gráfico 1. Regressão linear de absorvâncias por diferentes concentrações entre mangas de diferentes variedades e estados de maturação, Teresina, PI, 2018.

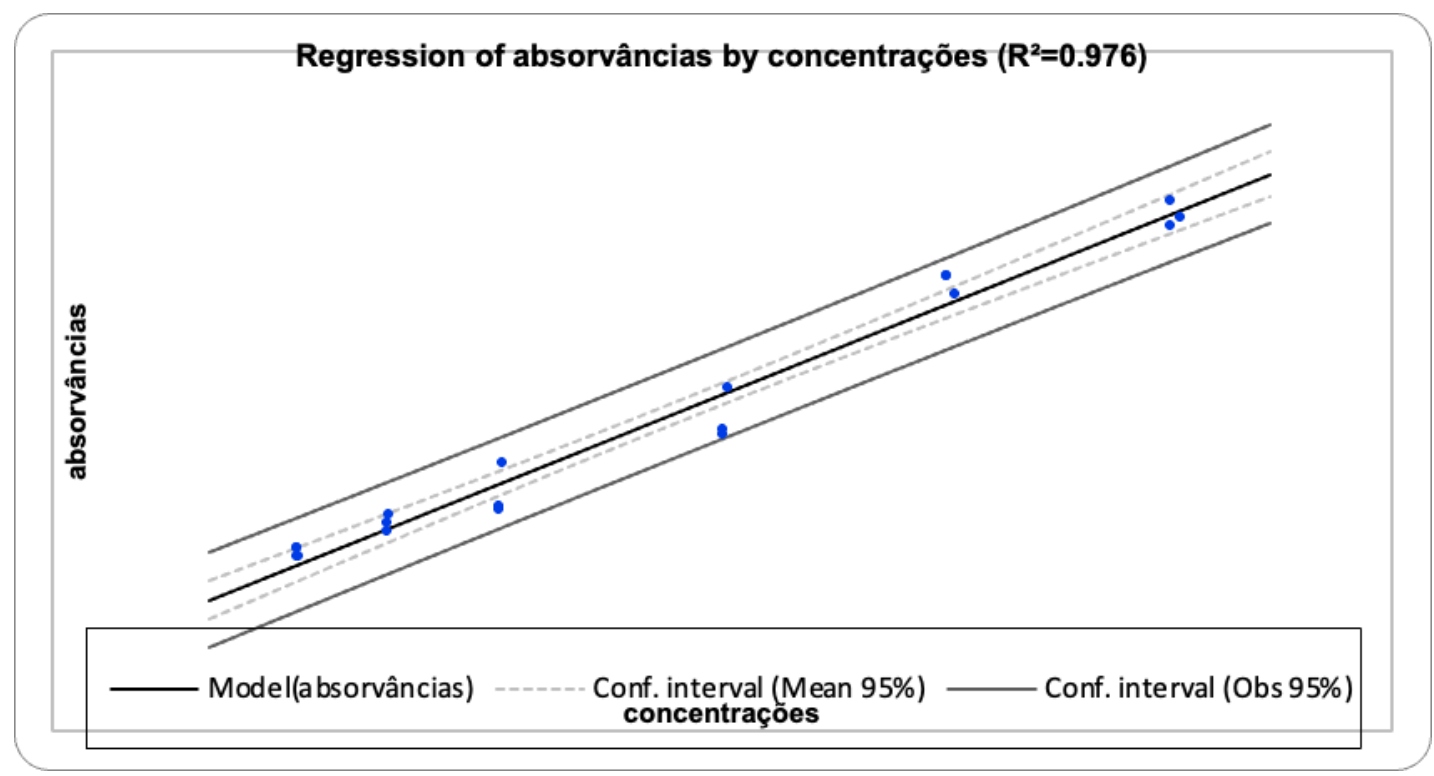

*Teste estatístico xlstat. Fonte: Dados da pesquisa (2018).

Como se observa no Gráfico 1 sobre regressão linear de absorvâncias por diferentes concentrações, o valor do $\mathrm{R}^{2}=$ 0.976 é bom, ou seja, o resultado foi estatisticamente significativo, pois houve boa linearidade, já que quanto mais os pontos se aproximam da linha central, melhor foi a análise, o R² é uma medida da qualidade da regressão, ou seja, quanto mais próximo de 1 , mais confiável é para determinar as concentrações.

A partir do gráfico 1 de regressão linear foi desenvolvido uma fórmula matemática que permita estimar qualquer concentração no intervalo que foi utilizado, neste caso foi de 2 até 0 , pode usar a fórmula para determinar a concentração de glicose com base na absorvância.

Absorvância $=-2.84550359693727 \mathrm{E}-02+0.42157453188871 *$ concentrações 
A linearidade de um método analítico corresponde a sua característica de responder proporcionalmente à adição da substância em estudo em uma dada faixa de concentração. Este parâmetro foi avaliado utilizando-se os coeficientes de correlação linear das curvas de calibração adotadas para o estudo da seletividade. De acordo com o mesmo estudo o método

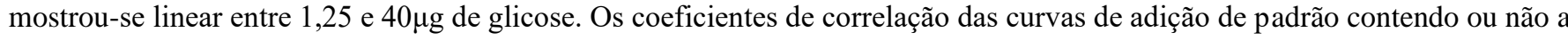
matriz da amostra (0,9981 e 0,9944, respectivamente) são considerados adequados, pois estão acima de 0,90. A estimativa desse coeficiente assegura a qualidade da curva obtida, pois valores próximos a um indicam uma menor dispersão do conjunto de pontos experimentais e menor incerteza dos coeficientes de regressão estimados (Pereira et al., 2008).

A pesquisa foi realizada utilizando-se dois métodos para determinação de glicose em diferentes variedades de manga nos diferentes estados de maturação, que foram o kit para glicose sanguínea pela análise direta e por curva analítica. A tabela 2 a seguir mostra uma comparação entre estes métodos nas mangas e qual deles se mostrou mais eficaz para determinar a glicose.

Tabela 2. Comparação dos teores de glicose presentes nas variedades verdes e maduras, determinadas usando kit para glicose sanguínea pela análise direta e por curva analítica, Teresina - PI, 2018.

Variedades

$\begin{array}{ccccc} & \text { Verde Kit } & \text { Verde Curva } & \text { Madura Kit } & \text { Madura Curva } \\ \text { Palmer } & 55.42 \pm 26.95 & 33.35 \pm 12.93 & 87.86 \pm 20.95 & 55.95 \pm 12.73 \\ \text { Tommy Atkins } & 63.72 \pm 60.07 & 34.15 \pm 25.83 & 104.3 \pm 32.58 & 45.35 \pm 12.05 \\ \text { Rosa } & 182.5 \pm 10.85 & 94.35 \pm 10.85 & 301.3 \pm 101.4 & 142.3 \pm 45.63\end{array}$

$* \pm$ desvio padrão, teste estatístico ANOVA. Fonte: Dados da pesquisa (2017).

Observa-se na tabela 2 uma comparação entre o kit para determinação de glicose e a curva de calibração analítica, que são métodos de determinação de glicose que foram utilizados entre diferentes variedades e estados de maturação. O método que expressou um resultado maior e eficaz para determiná-la foi o kit para determinação de glicose em todas as variedades de manga e estado de maturação.

A manga verde, que teve uma maior quantidade de glicose foi a Rosa, com 182.5 (Kit) e 94.35 (Curva). Em relação à manga madura, a que obteve maior quantidade de glicose também foi a Rosa com 301.3 (Kit) e 142.3 (Curva). A manga verde que teve uma menor quantidade de glicose foi a Palmer com 55.42 (Kit) e 33.35 (Curva). Na manga madura, a que obteve menor quantidade de glicose foi a Palmer com 87.86 (Kit) e Tommy com 45.35 (Curva).

O Gráfico 2 a seguir demonstra uma pesquisa realizada na literatura acerca dos valores de glicose e frutose em 44 diferentes frutas, para saber se existe ou não correlação. 
Gráfico 2. Curva de regressão linear demonstrando a correlação entre valores de glicose e frutose em frutas, Teresina, PI, 2018.

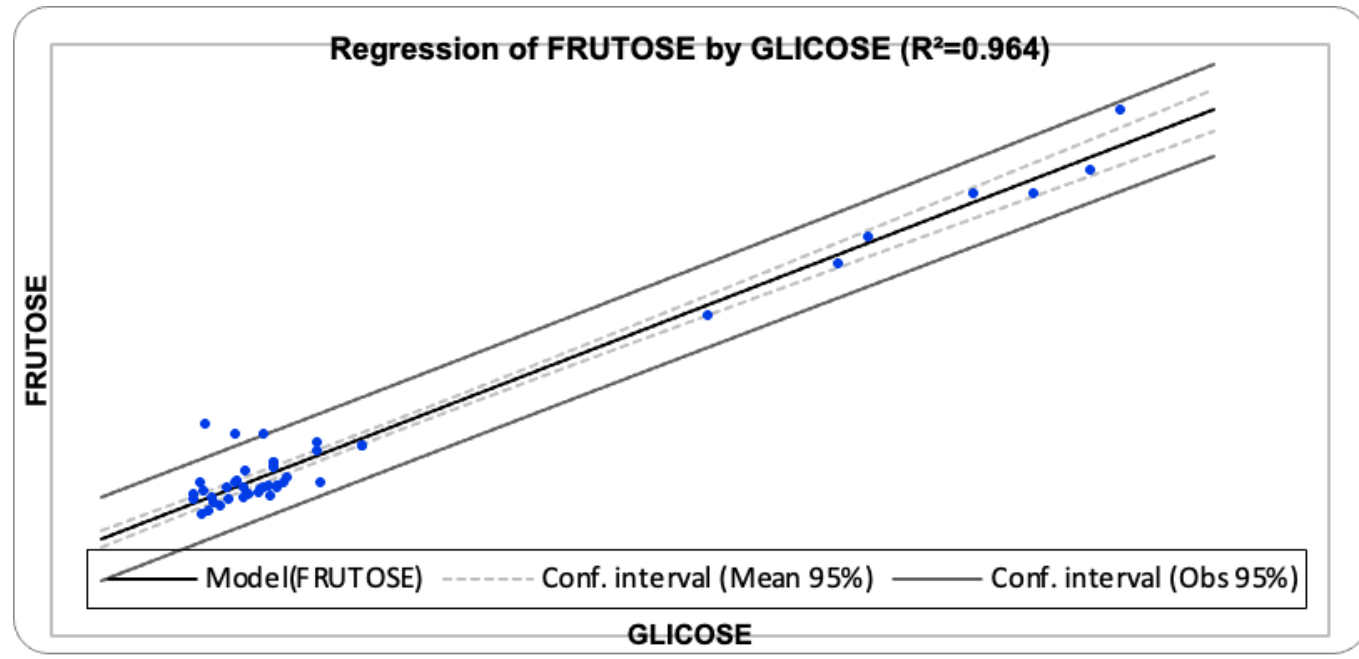

*teste estatístico xlstat. Fonte: Dados da pesquisa (2018).

O Gráfico 2 representa a curva de regressão linear de glicose e frutose em diferentes frutas, no qual é possível prevê o comportamento da relação glicose/frutose com 96,4\%, quando estima a frutose a partir da glicose apresentou excelente linearidade, pois o $\mathrm{r}^{2}$ está acima de 0,95, ou seja, quanto mais próximo o valor de 1,00 melhor. A partir deste gráfico pode-se perceber que a frutose pode ser estimada e expressa a partir do valor da glicose encontrada, uma vez aplicada na fórmula criada a seguir e demostrada no gráfico 3:

$$
\text { Frutose }=1.18369668900434+0.924568750228745 * \text { GLICOSE }
$$

Gráfico 3. Níveis de frutose estimados pela equação da reta de correlação entre os níveis de glicose e frutose nas diferentes condições das variedades das mangas, Teresina - PI, 2018.

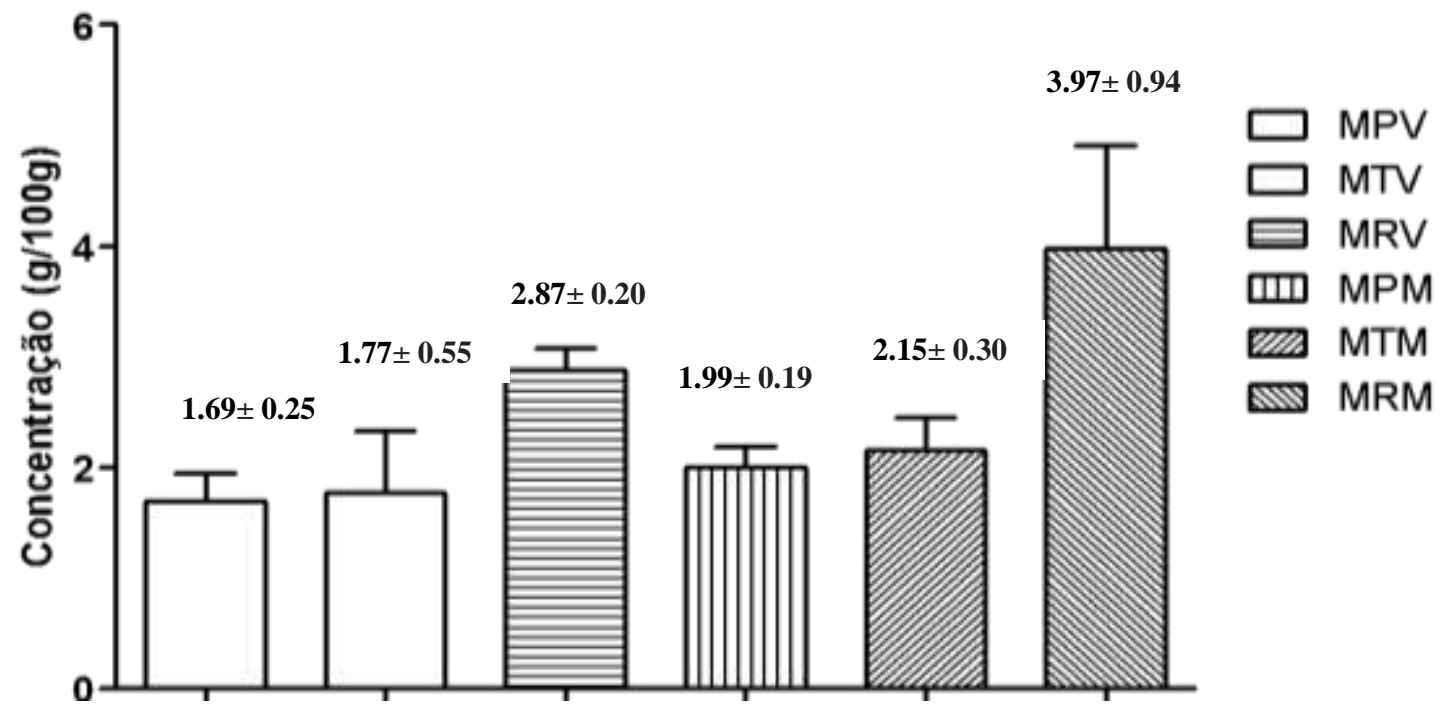

*MPV= manga palmer verde, $\mathrm{MTV}=$ manga tommy verde, $\mathrm{MRV}=$ manga rosa verde, $\mathrm{MPM}=$ manga palmer madura, $\mathrm{MTM}=$ manga tommy madura, $\mathrm{MRM}=$ manga rosa madura. Teste estatístico ANOVA. $\mathrm{p}=0.0084$. Fonte: Dados da pesquisa (2018). 
O Gráfico 4 está representando os níveis de frutose estimados pela equação da reta de correlação entre os níveis de glicose e frutose nas diferentes condições das variedades de manga expresso pela curva de regressão linear no gráfico 2 e 3 . Os níveis de frutose estimados nas três variedades de manga apresentaram diferenças estatisticamente significativas ( $\mathrm{p}=0.0084$ ) quando comparadas com a variedade Rosa, pois esta apresentou valores de frutose superiores ao de todas as outras variedades, tanto na manga verde, como madura, e mesmo comparando a variedade Rosa verde e madura, a madura apresentou quantidade de frutose superior. A frutose foi o açúcar predominante na manga Tommy Atkins e Palmer, no qual foram obtidas de um produtor comercial localizado em Joanópolis (SP) (Bernardes-Silva, Lajolo, Cordenunsi, 2003).

A frutose, por ser de baixo índice glicêmico (IG), é um carboidrato de absorção lenta muito utilizada na indústria alimentícia, que oferta produtos que aumentam a saciedade e tem menor IG. A frutose ou levulose é o mais doce de todos os açúcares naturais ou de mesa. Encontrada, principalmente, em frutas e mel, pode apresentar alguma redução calórica na aplicação final. No Brasil, todavia, não é permitida a adição em alimentos e bebidas para dietas com restrição de açúcares, uma vez que a legislação permite o limite máximo de 0,5 gramas da soma de glicose, sacarose e frutose por $100 \mathrm{~g} / \mathrm{ml}$ dos alimentos prontos para o consumo. Porém, a ingestão de frutose deve ser evitada em pacientes diabéticos, pois seu uso pode provocar hiperglicemia (Silva et al., 2017). Além disso, a frutose é o monossacarídeo predominante em várias frutas, incluindo maçãs, laranjas e melões.

\subsection{Umidade}

A umidade de um alimento está relacionada com sua estabilidade, qualidade e composição, podendo afetar a estocagem, a embalagem e o processamento. Corresponde à perda de peso do produto quando aquecido em condições nas quais a água é removida. Sua determinação possui grande importância, sendo ponto de partida na análise dos alimentos por refletir na sua perecibilidade, estabilidade econômica e preservação, pois quando não estão dentro das recomendações resultam em deterioração microbiológica e qualidade sensorial geral da fruta, como a aparência, cor, textura e sabor, e estão demonstradas na tabela 3 (Mendes-Filho, Carvalho, Souza, 2014).

Tabela 3. valores médios e desvio padrão de umidade entre mangas de diferentes variedades e estados de maturação, Teresina, PI, 2017.

\begin{tabular}{ccc}
\hline Variedades & \multicolumn{2}{c}{ Maturação das frutas } \\
& ${ }^{*}$ Verde & ${ }^{*}$ Madura \\
Palmer & $85.95 \% \pm 2.06$ & $79.28 \% \pm 3.14$ \\
Tommy Atkins & $83.76 \% \pm 6.71$ & $79.12 \% \pm 4.16$ \\
Rosa & $83.5 \% \pm 1.63$ & $79.7 \% \pm 1.21$ \\
& &
\end{tabular}

Teste estatístico ANOVA. ${ }^{\#}(\mathrm{p}>0,05)$. Fonte: Dados da pesquisa (2017).

$\mathrm{Na}$ Tabela 3 não se observou diferenças estatisticamente significativas ( $p>0,05$ ) entre as diferentes amostras analisadas, pois não houve grande variabilidade entre as médias das diferentes variedades.

As amostras da polpa da manga Tommy apresentaram teor de umidade de $83,76 \%$ de água nos frutos verde e 79,12\% nos maduros. O maior constituinte da composição da manga Tommy foi à umidade, que correspondeu a 82,11\% podendo contribuir para a perecibilidade do fruto depois de colhido e se manuseado inadequadamente (Marques et al., 2010). 
Os valores obtidos para parâmetro de umidade da manga Rosa variaram entre 83,5\% verde e 79,7\% madura. Em estudo semelhante obteve uma média de 79,05\% (Leal, 2016).

As amostras da manga Palmer apresentaram teor de umidade de $85,95 \%$ nos frutos verde e 79,28\% nos maduros. Segundo dados da literatura o valor de umidade da manga Palmer é 79,70\%. Na determinação de umidade em análise físico química a polpa da manga, apresentou valores de umidade de 85,74\% (Rocha, 2017).

O comportamento habitual dos frutos, em relação ao teor de umidade preconiza achar-se a água acumulada em maiores proporções no fruto verde, e, conforme este vai amadurecendo, o nível tende a decrescer devido á transpiração, que é mais intensa nesse período (Maciel, 2009).

Neste estudo observou-se de fato uma redução dos níveis de umidade assim como citam os autores acima, ou seja, com o processo de amadurecimento a manga perde um pouco do seu conteúdo de água, porém as diferenças não foram estatisticamente significativas e não podem desta forma, explicar as diferenças nos níveis de glicose livre.

Segundo Souza et al., (2020), avaliou as médias de umidade, observou as porcentagens de 76\%, 66\%, 80\% e 79\%, para as frutas abacaxi, banana, caju e manga, respectivamente. Fatores físico-químicos como o pH e a umidade alteram de formam direta a qualidade sensorial das frutas.

Com o avanço da maturação houve aumento no teor de água e açúcares na polpa da manga e relação ao teor de vitamina C, houve uma redução significativa durante a maturação (Costa et al., 2019).

A determinação do potencial hiperglicêmico e do índice glicêmico é um procedimento experimental que deve ser feito com cada alimento para que ele possa ser determinado, pois não é possível realizar o cálculo de índice glicêmico com base nos valores de glicose.

O potencial hiperglicêmico que existe nas tabelas da literatura foram feitas com variedades cultivadas fora do Brasil (Ásia e Oceania), ou seja apesar de ser da mesma espécie, as variedades cultivadas no mesmo país apresentam diferenças significativas no teor de $\mathrm{CHO}$, assim sendo existe a necessidade para determinação do IG para cada cultivar de cada país, pois as variedades variam entre as concentrações de glicose.

\section{Conclusão}

As variedades de manga testada apresentaram variações significativas nos teores de carboidrato de baixo peso molecular especialmente a variedade Rosa. A manga Palmer (verde e madura) apresentou menor teor de açúcares de baixo peso molecular. A manga Rosa (verde e madura) apresentou maior teor de açúcares de baixo peso molecular. As diferenças entre as variedades, conforme o resultado de concentração de glicose, foram significativas.

$\mathrm{O}$ método de determinação de umidade em balança infravermelho é eficiente e diminui com o amadurecimento, mas não foi significativo. Todas as variedades encontram-se dentro da faixa recomendada para umidade. A manga Palmer verde apresentou menor potencial para elevar a glicemia devido à presença de menor nível de carboidrato. Apresentou maior quantidade de glicose a manga Rosa madura, com maior potencial para elevar a glicemia devido à presença de maior nível de carboidrato.

O método enzimático desenvolvido demonstrou-se capaz para a quantificação espectrofotométrica do teor de glicose em amostras de mangas. Além disso, a frutose pode ser estimada e expressa a partir do valor da glicose encontrada, uma vez aplicada na fórmula criada.

É necessário a determinação do índice glicêmico para as variedades de frutas no Brasil, uma vez que diferentes variedades produzidas nas mesmas regiões apresentam diferenças significativa nos teores de carboidrato. Pode-se sugerir a 
análise de uma quantidade maior de amostras e variedades de mangas, em outros estágios de maturação para favorecer as informações nutricionais.

\section{Referências}

Bezerra, T. S., Costa, J. M. C., Afonso, M. R. A., Maia, G. A., \& Clemente, A. (2011). Avaliação físico-química e aplicação de modelos matemáticos na predição do comportamento de polpas de manga desidratadas em pó. Revista Ceres, Viçosa, 58 (3)

Braz, V. B., Nunes, E.S., Vieira, G., Júnior, J. I. R., Bertini, L A. \& Couto, F. A. A. (2008). Indução do amadurecimento de mangas cv. Tommy Atkins e CV. Ubá pela aplicação de ethephon pós-colheita. Bragantia. Campinas, 67(1) 225-32.

Caldas, B. S., Constantino, L. V., Silva, C. H. G. A., Madeira, T. B., \& Nixdorf, S.L (2015). Determinação de açúcares em suco concentrado e néctar de uva: comparativo empregando refratometria, espectrofotometria e cromatografia líquida. Scientia Chromatographica, 7(1), 53-63.

Carvalho, G. G., \& Alfenas, R. C. G. (2008). Índice glicêmico: uma abordagem crítica acerca de sua utilização na prevenção e no tratamento de fatores de risco cardiovasculares. Revista Nutri, Campinas, 577-587.

Costa, M. S., Almeida, F. A. C., Coelho, B. E. S., Josenara Daiane de Souza Costa, J. D. S., \& Figueiredo Neto, A. (2019). Composição química da polpa de manga 'Ataulfo' em diferentes estádios de maturação. Journal of Environmental Analysis and Progress, 5 (1), 11-16.

Filho, N. E. M., Carvalho, M. P. C., \& Souza, J. M. T. (2014). Determinação de macrocomponentes e nutrientes minerais da polpa de manga (Mangifera indica L.). Revista Perspectivas da Ciência e Tecnologia, 6(2).

Fontelles, M.J; Simões, M.G.; Farias, S.H.; Fontelles, R.G.S. Metodologia da pesquisa científica: diretrizes para a elaboração de um protocolo de pesquisa/ Scientific research methodology: guidelines for elaboration of a research protocol. Rev. Para. Med. = Rev. Para. Med. (Impr.); 23(3), jul.-set. 2009.

Gaino, N. M., \& Silva M. V. Consumo de frutose e impacto na saúde humana. (2011). Segurança Alimentar e Nutricional, Campinas, 18 (2), 88-98.

Ganogpichayagrai, A., Palanuvej, C., \& Ruangrungsi, N. (2017). Antidiabetic and Anticancer activities of mangifera indica cv. Okrongleaves. J AdvPharmTechnol Res.

Leal, S. S. de. (2016). Determinação de micronutrientes na manga: variedades rosa e espada. [Monografia Química Licenciatura, Universidade Federal do Maranhão].

Lemos, K. H.; Patek, T. C. \& Mezzomo, T. R. (2017). Determinação do índice glicêmico e da carga glicêmica de dietas hospitalares servidas para diabéticos. Scientia Medica, Curitiba, 27(4).

Maia, M.C.C, Araújo, L. B., Dias, C.T.S., Oliveira, L.C., Vasconcelos, L.F. L., Carvalho Júnior, J. E. V., Simeão, M. \& Bastos, Y. G. M. (2016). Seleção de genótipos de mango rosa em utilizando o método multivariável-biplot. Revista.Ciência Rural, 46(10).

Marques A., Chicaybam, G., Araujo, M. T., Manhães , L. R. T., \& Sabaa-Srur, A. U. O. (2010). Composição centesimal e de minerais de casca e polpa de manga (mangifera indica 1.) Cv. Tommy atkins. Revista. Bras. Frutic. 32 (4), 1206-1210.

Maciel, L. F. de (2009). Caracterização físico-química e avaliação de compostos bioativos de mangas (Mangifera indica L.) proveniente de cultivo biodinâmico, orgânico e convencional. [Dissertação, Mestrado em Ciência de Alimentos, Universidade Federal da Bahia].

Massa, N. M. L. (2014). Concentrado de melancia (citrullus vulgaris schrad): aceitação sensorial, parâmetros microbiológicos, físico-químicos e determinação de fitonutrientes. B.CEPPA, 32(1), 113-124.

Melo, E. A., \& Araújo, C. R. (2011). Mangas das variedades espada, rosa e tommy atkins: compostos bioativos e potencial antioxidante. Semina: Ciências Agrárias, 32(4), 1451-1460.

Pereira, E. I. P., Emanuelli, T., Bisognin, D. A., \& Freitas, S. T. (2008). Otimização e validação de um método enzimático para a determinação de glicose em tubérculos de batata. Ciência Rural, 38(5), 1227-1232.

Rocha, F. O. (2017). Secagem de polpa de manga (Mangifera indica, L.) cv. Palmer em spray-dryer : condições de secagem e estabilidade. [Dissertação de mestrado, Universidade Federal do Ceará, Programa de Pós-Graduação em Ciência e Tecnologia de Alimentos. Fortaleza].

Santos, A. A. Dosagem de açúcares redutores com o reativo DNS em microplaca. (2017). Braz. J. Food Technol. Campinas, 20.

Silva-Bernardes P.F., Lajolo, M., \& Cordenunsi, B.R. (2003). Evolução dos teores de amido e açúcares solúveis durante o desenvolvimento e amadurecimento de diferentes cultivares de manga. Ciênc. Tecnol. Aliment, Campinas, 23, 116-120.

Silva, D. B., Leal, E. M., Santos, G. M., Oliveira, J. M. S., Saldanha, N. M. V. P., Sousa, P. V. L., Oliveira, K. K. S., Cavalcante, R. M. S., \& Barros, N. V. A. (2017). Caracterização dos efeitos da frutose na dieta de pacientes diabéticos. Braz.J. Surg.Clin. Res. 20(2), 139-145.

Silva, A.C., Souza, A. P., Leonel, S., Souza, M. E. \& Tanaka, A. A. (2012). Caracterização e correlação física e química dos frutos de cultivares de mangueira em São Manuel, São Paulo. Magistra, 24(1), 15 - 26.

Silva, K. C., Nobre, L. N., Vicente, S. E. C. F., Moreira, L. L., Lessa, A. C., \& Lamounier, J. A.(2016). Influência do índice glicêmico e carga glicêmica da dieta sobre o risco de sobrepeso e adiposidade na infância. Revista Paul Pediatr, 3(34), 293-300.

Souza, I. C.C., Carvalho, A. C. B., Silva Neto, J. M. D., Fernandes, J. P. C., Rocha Junior, J. D., Araújo, F. M. M. C., \& Melo, R. L. F. (2020). Caracterização físico-química dos frutos tropicais do Nordeste brasileiro. Research, Society and Development, 9 (6). 